\title{
Not Only I: Notes on the Syntax of Focus Binding
}

\section{Tom Roeper}

\section{Introduction}

Heim (1991) has brought attention to the role of indexicals in sentences like (1):

(1) Only I got a question that I could answer.

Partee (1989) noted a similar effect for sentences like (2):

(2) I'm the only one around here who will admit that I might be wrong.

In (2), the indexical binding has to proceed via a pronominal the only one that leads into a wh-relative clause. Kratzer $(1998,2006)$ has explored a variety of morphological variations on how these indexicals interact and exhibit agreement. In informal notes (Roeper 1999), I observed that proper nouns can also participate in this form of binding:

(3) Only Mary still looks like Mary in these old pictures.

In what follows we will describe, in syntactic terms, a variety of contexts where this connection both succeeds and fails, without specifying exactly how semantic representations might be involved, but rather observing from the other side of the interface what syntactic dimensions are engaged.

\subsection{Semantics}

First, it should be observed that whatever semantics are used here (structured meanings and alternative semantics have been explored), the effects must capture cases like (4):

(4) Even I got a question that I couldn't answer.

Here the same binding occurs, but the determination of the relevant set entails whatever value scale is implied by the word even.

\subsection{Delicate judgments and a deeper method}

Second, we would like to freely observe that, while the core intuitions about proper nouns are "delicate", in current parlance, they move in a common direction. Thus while one may not always be confident in some judgments, one can be confident in the 
method of searching through variation to see if it takes a common direction. In particular, many grammaticality judgments become clearer once a larger array of relevant facts are brought into focus. For instance, VP-ellipsis judgments usually become more liberal as the sentence becomes more complex, cf. (5).

(5) A lot of this material can be presented in an informal and accessible fashion, and often I do.

(Hardt (to appear), quotation from an interview with Noam Chomsky)

In comparison, a shorter version seems decidedly worse:

(6) *? A lot of this material can be presented, and often I do.

The only Mary-cases like (3) by contrast become systematically worse, not better, in complex environments, as we shall show. Therefore, the collective data points to an elaboration of what constitutes an "intervenor", what makes a sentence complex, in the sense that has become common in describing syntactic barriers.

\section{The breadth of the phenomenon}

What constituent categories allow set-induction? We argue that all categories do. Consider now the variety of proper nouns which, in a suitable context, allow setinduction:

(7) Proper names:

a. In these dim photos, only Mary still looks like Mary.

The primary reading here is that therefore, Bill does not look like Bill anymore in the mentioned photos - nobody except Mary looks like themselves anymore in them. Likewise we have:

(8) a. Only Mary can act like Mary in the culture of Greenwich village (noone else can act like themselves there).

b. Only Mary can act like Mary in a foreign culture (the rest of us can't really be ourselves).

c. Only Fred still talks like Fred in front of royalty (everyone else puts on a phony accent).

(9) Other proper nouns:

a. Only Mt Hood still looks like Mt Hood even in winter (no other mountain looks like itself in winter).

b. Only Christmas is always like Christmas in every culture (no other festivity is always the same in every culture).

c. Only my birthday makes me feel like my birthday in my old hometown (Christmas does not feel like Christmas in my old hometown). 
(10) Indefinites:

a. Only a very odd person still looks like a very odd person after using modern make-up techniques (ordinary people don't look ordinary).

b. Only a very loud person sounds like a loud person in the old radio tapes (other accents don't come through as themselves).

c. Only a very very tall person looks like a very very tall person from a great distance (small people don't look like small people, they just look normal).

(11) Definites:

In these old pictures, only the living room still looks like the living room (no other room looks like itself).

Other kinds of indexicals, which carry a point of view (henceforth: POV), also seem to allow this behavior:

(12) Point of view (POV) indexicals:

a. Only now can one appreciate now (then one could not appreciate then or then one could not appreciate now).

b. Only here can one praise here (there one cannot praise there).

It seems to be a truly free phenomenon, applicable to VP gerunds as well:

(13) a. Only ballet dancing looks like ballet dancing in a still photo (breakdance doesn't look like breakdance, squaredance doesn't look like squaredance... in a still photo).

b. Only being nice is appreciated as being nice (acting any other way is misinterpreted).

If these examples are sustainable, then it is clear that the element which can "associate with focus" is not limited to a set of lexical types (like pronouns) or syntactic types, or specificity types. To buttress out this view, we undertook an informal experiment in a linguistics class, and asked undergraduates to complete sentence (14).

(14) Only Mary looks like Mary, no one else....

14 out of 18 undergraduates gave as an answer: looks like themselves rather than looks like Mary. This showed that the interpretation is readily available and not an obscure alternative.

\section{Divergence}

Where do these structures begin to diverge? Notice that we get a sharp degradation when we substitute person for the pronoun in the Partee example:

(15) ?? I am the only person around here who will admit that I might be wrong.

The pragmatics of the intended meaning is so strong that no other meaning is plausible; why should a person other than me be caused to admit something about me. Yet, the 
grammaticality seems to decline, which suggests structural factors interfering with our intuitive sense of well-formedness. Notably we find that where the non-set reading is plausible it will take over easily:

(16) I am the only person around here who thinks that I might be wrong.

This has the reading that other people might think that I am wrong far more naturally than the reading that they might be wrong. While only I can form a chain with the only one who, it seems that proper nouns do not so readily accept that connection:

(17) ?? In this picture, Mary is the only one who looks like Mary, no one else does.

Anything other than one leads to much stronger ungrammaticality:

(18) a. ?* Only Mt Hood is a mountain that looks like Mt Hood.

b. ?* In these old photos, only Ana is wearing a dress that makes Ana look good (everyone else...)

It is not clear that an equivalent to one for location exists, so that place does not allow us to build a chain between the two instances of the proper name:

(19) * Only Mt Hood is a place that retains the majesty of Mt Hood in all weather.

These examples suggest that indexicals more readily agree with a pronoun one than with proper names, which is not surprising since they are also pronouns. It implies that more than co-indexing is involved, namely a whole array of features, including [pronominal] for all types of constituents and perhaps all types of binding. ${ }^{1}$

\section{Association with focus}

Now we can begin to ask what the best explanatory tools will be. Rooth (1992) describes the phenomenon of association with focus where non-identical elements are linked, cf. (20):

(20) a. Only John wears TWO hats every day.

This is commonly described as a leftward movement at LF of the focal element, very roughly as (20'), where both someone and $x$ have the status of traces/copied elements.

(20’) [Only John, TWO [someone is wearing x-number hats]]

Notably this association with focus can occur across typical barriers:

(20) b. Only John knows someone who wears TWO hats every day.

It is generally assumed that the apparatus for focus binding is not within a movement theory if it does not obey obvious barriers. We will simply sketch an approach to the

1 Kratzer (2006) pursues the features behind agreement in these contexts in a paper "Minimal Pronouns" presented in Saarbrücken, which follows Kratzer (1998). 
possibility of extraction from islands that continues to assume the presence of real movement. There have been many claims that subjacency is a weak effect and therefore it is not very unusual to seek a method to circumvent islands rather than abandon movement. We will discuss alternatives briefly below.

First, it is known that some quantifiers, like each, are also able to scope out of islands [e.g. there is some fact that bothers each man]. Terada (2003) has made the interesting argument that each has both an anaphoric feature and a distributive feature to account for the fact that it is linked to the subject but confers distributivity on an adjective (cf. 21a,c).

(21) a. The boys arrived each alone.

b.* The boys arrived each together.

c. The boys arrived each together with his mother.

In (21b) distributivity fails, but is restored in (21c). We can carry the idea over to the only phenomenon. Let us assume, unlike for wh-words that are attracted to an invisible [+wh] feature, a link to a visible only creates an anaphoric connection. However, compositional interpretation of TWO requires movement to an LF adjacent position. We will not explore this idea further, but suggest that it may be an avenue through which one can explain the evasion of islandhood. Thus we will assume both that leftward movement occurs and that an anaphoric binding relation is present. We can capture that additional feature for all our data if we assume a rule that establishes binding under phonetic and semantic identity:

Binding: 1. Coindex identical words in a c-command domain.

2. At LF, move focal material to a position accessible to the only-operator.

3. Treat identical foci as bound variables.

Now we need to define the movement path in such a way as to permit intervention.

\subsection{C-command}

It is clear that c-command is a requirement for the set-induction cases (as pointed out to me by R. Bhatt (pc)):

\section{(23) Only my daughter helped me, Jane's daughter didn't...}

For (23), one might marginally obtain set-induction in the first clause, but it does not continue into the elided VP: Jane's daughter didn't help me, not Jane didn't help me. This is predicted if c-command is a requirement on the binding relation. Given ccommand, if we assume that leftward movement underlies the bound focus in all instances of binding, then the LF analysis of focus movement for these phrases suggests that we should expect barrier effects for movement. 


\subsection{Perspectivals}

External perspective adverbs block long-distance movement:

(24) *How did John say $t_{\text {how }}$ that he unfortunately could win the game $t_{\text {how. }}$

Adverbs like unfortunately, luckily or certainly all imply an outside perspective, which Speas (1999) calls the "Seat of Knowledge". Potts (2005) discusses expressives like damn or sure and related terms which he argues can be interpreted at the root of the clause, but require what he calls a multi-dimensional semantic representation. This translates into the claim that the expressive features percolate to the $\mathrm{CP}$ of the clause. They also appear to shift POV to a speaker. We find that these outside perspectives weakly block set-induction (cf. (25)), and that expressives also block it (cf. (26)).

(25) a. ?? Only I unfortunately got a question that I could answer.

b. ?? Only Mary unfortunately got a question that Mary could answer.

Expressives also block:

(26) a. ?? Only Mary sure looks like Mary in these old pictures.

b. ??? Only I sure got a question that I could answer.

Again the reading remains the same, but the judgment of grammaticality seems to decline. Ultimately, we need to explain exactly how the movement chain experiences each kind of interruption.

\subsection{Point of view (POV)}

It is commonly assumed external perspective adverbs reflect a speaker POV, but a more careful look indicates that they imply a general POV. Sentences like Unfortunately, Bush is president are assertions that it is unfortunate that Bush is president as a general proposition, not as a claim from a speaker POV comparable to deictics and indexicals. It is not immediately clear why such elements should function as intervenors. While indexicals like $I$, you, we seem to all have the speaker POV, we suggest here that they each reflect slightly different POVs, as do expressives. That is, an expression like we actually assumes a shift in perspective from $I$. In addition, I seem to me suggests two guises, and an expressive like Wow, I did it assumes a slightly different perspective for wow than for $I$. The strongest hypothesis then is that (27) holds.

(27) Every perspectival shift can interfere with movement.

Before we hazard some possible proposals, we need to see if other phenomena fall into line. 


\subsection{Expletives}

Consider other forms of coreference:

(28) a. ?Only John thinks John can do it (no one else thinks they can do it).

b. *Only John thinks that the bastard can do it (no one else thinks they can do it).

Here we cannot obtain a reading where there are a set of bastards each of whom thinks of himself that he can do it. Note again that the outside perspective that identifies John seems a little less particularized than the one that labels him a bastard.

\subsection{Sequence of tense}

It has been noted that Sequence of Tense can affect binding as well, and therefore could affect extraction. Consider (29):

(29) a. Every boy thought he could do it.

b. Every boy thought he can do it.

The (b) example (following Nunes and Thompson 1995) favors the external reading over the bound reading. We find the same to be true of the cases in (39), where if the tense contrast is clear, the focal binding fails:

(30) a. Only Mary thought Mary IS big.

b. Only I thought that I AM big.

c. Only I got a question that I CAN answer.

While the pragmatics may force the bound variable reading through, speakers uniformly find the sentence "worse".

\subsection{Indexicals against indexicals: experiencer datives}

While the sentence in (31) seems fine, the judgment degrades sharply when another indexical intervenes, cf. (32).

(31) Only you seem to think you can do it.

(32) Only you seem to me to think you can do it,

a. no one else [thinks you can do it]

b. ?* no one else [thinks they can do it]

Note that the (a) example has a default interpretation of "seems to everyone in general", which is then shifted to the speaker point of view with the addition to me. This observation is modeled on Brandt's (2006) work on cipient datives in German where he argues that a separate truth interval is called for with the productively available cipient 
datives in German. Predictably, under our refined view of perspectives, even seemingly identical indexicals cause a problem:

(33) ?? Only I seem to me to get my homework done.

Here again we suggest that two guises are present: I see myself as if from outside. Therefore, it may still be that two different POVs are present.

Our goal is to formulate the syntactic dimension that would reflect this semantic interface with the least complex point of interaction between syntax and semantics, that is, the impact of the semantic representation would ideally be encapsulated in an efficiently viable form. We suggest that (34) and (35) hold:

(34) There exists a POV feature in a Spec position with an agreement index linked to its head constituent.

(35) There exists a POV for a general default (seat of knowledge) assumption, thereby allowing transition of movement through it if the moved element has a compatible index.

Therefore, if there is a contrary POV feature present, it will block movement.

\subsection{Non-restrictive relatives and late merge}

Finally, let us consider a rather subtle contrast which has been the source of arguments for late merge. Pairs like the following call for a contrastive judgment:

(36) a. Only I got a question that I could answer (no one else got a question they could answer).

b. Only I got a question which I could answer (no one else got a question).

The (b) example seems to break the connection again, though judgments can be confused by the overwhelming pragmatics favoring binding.

\subsection{Summary}

We have found five contexts where there seems to be intervenors that block association with focus:

I Late merged relative clauses

II Sequence of tense contexts

III Expletive expressives

IV Adverbial outside perspectives

$\mathrm{V}$ Dative indexicals

It is not necessarily the case that these cases deserve a unified explanation. We will take a few steps in that direction nonetheless. 


\section{Explanatory levels}

The kinds of phenomena we have been examining have been largely the concern of those projecting semantic models (e.g. Potts 2005, Schlenker 2003, Nevins and Pranand 2002). Our approach will be uncommitted about what kind of representation captures the meaning differences best. Instead, we argue that whatever semantic approach is taken, it must have a syntactic projection such that there are computable syntactic consequences. This is not the only possible position. One could argue that all of the sentences we have considered are syntactically well-formed and their deviation lies wholly in a semantic realm.

If, however, they pattern together with known syntactic variation, then a common representational level is called for. In this brief paper we will sketch the lines of an account with many details omitted. A vision of the overarching architecture of explanation should be useful in itself in giving a full picture of the set-induction phenomena.

\subsection{The relative clause case}

The relative clause case is at first puzzling: why should a restrictive that-clause tolerate binding, while a non-restrictive which-clause does not? We can utilize the focus theory if, first, we assume that non-restrictive relative which-clauses are merged late, as argued by Bhatt and Pancheva (2004), following Lebeaux's theory of late merge. Then we can argue that late merge is high in the tree, attached at the root CP and that therefore, the cyclic movement path is not formulable. This restriction is then reducible to the failure of c-command.

\subsection{The Relativized Minimality option}

One approach to intervention data would be to argue that the traditional mode of intervention is at work:

sure $=$ a positive item like negation

unfortunately $=$ an adverb

Each of these are blocked by Relativized Minimality since adverbs are blockers for adjunct phrases. We can, at a subtle level, see an effect for negation as well. The following sentences provide a contrast:

(37) a. Only I got a question that I couldn't answer.

b. ?Only I didn't get a question that I could answer.

The bound reading comes through for the (b) example, but it seems less acceptable than in the (a) example. Under a movement analysis, the focal element passes through the NegP node in the (b) example. However, Relativized Minimality turns on the 
argument/adjunct contrast. It seems like different features are primarily involved in blocking in the full range of cases. Instead, we think a related approach would offer a better route toward building a successful interface with the semantic generalizations that are evident here, while preserving Rizzi's suggestion that unlike elements of the same type can interfere with each other. Our goal will be to treat as syntactic features as few of the semantic features as possible. Our examples all show the impact of perspective:

$\begin{array}{lll}\begin{array}{l}\text { indexicals } \\ \text { expressives }\end{array} & \Rightarrow & \text { speaker perspective } \\ \text { datives } & \Rightarrow & \text { speaker perspective } \\ \text { adverbs } & \Rightarrow & \text { seat of knowledge perspective } \\ \text { sequence of tense } & \Rightarrow & \text { seat of knowledge perspective }\end{array}$

From these we can argue that time, location, and person features are derivative, possibly within a system of implicatures, from what we can call a formal POV perspective which implies not only a person, but also a time and a location. However, formally we aim to have a simple POV index which conflicts with any non-identical POV index. We are deliberately both vague and restrictive. These features can obviously form a part of world variables and other semantic terms, but our goal is to find an encapsulated formulation that is exclusively relevant to to an efficient syntax. We call it "POVExternal" as distinct from "Person-POV" and "Subject-POV". It includes small perspectival shifts, new guises that arise as implicatures in sentences like I seem to me to be more prepared (one of 705 such examples from Google) as distinct from I seem to myself to be more prepared.

\subsection{Feature checking}

In that vein, let us assume, following Hollebrandse (2000), Speas (2004) Tenny and Speas (1998) and (in part) Nevins and Anand (2002), that there is a POV operator that can occupy the Spec position of a CP, DP, ADVP. and VP. This feature is projected in the Spec position of the DP, ADVP or CP, and it can percolate to the relevant Spec of each maximal projection that focus movement passes through. If POV operators occupy a Spec position, then they will constitute positions through which the Focus element must move to associate with only at LF. Let us further assume that the POV feature functions as a [+interpretable] feature which can be valued by the head feature it is in agreement with and can be repeatedly checked by features that pass through that node. Theoretical work in the minimalist tradition has not been completely explicit about what happens to the Feature-checking mechanism when information passes through repeated CPs or other phases in long-distance rules. In a quite detailed account, Hornstein, Nunes, and Grohmann (2005:295) argue that [+interpretable] phi-features are repeatedly checked in the Spec of participles and in Spec of $\mathrm{T}$ in passive constructions. ${ }^{2}$ The POV

2 "The interesting point for our discussion is that the phi-features of as alunas must enter into a checking relation with both the participial $\mathrm{T}$ and the matrix $\mathrm{T}$. This multiple checking thus confirms our previous conclusion that the phi-features of arguments are [+interpretable].” (p. 295) 
feature, including Person, is a natural extension of the phi-feature type which, like traditional phi-features, can be spelled out on other categories besides nouns (determiners, adjectives, etc).

We will assume that a focal phrase must move to have its features ultimately checked in the highest position where only also moves. In a sense, the POV feature is a "free rider", which must meet a compatibility requirement -- agreement -- with every intermediate node that is passed through.

We now make the prediction that only elements introducing an external perspective will interfere with set induction. We find our judgements to be weak, but they nonetheless proceed in the right direction: adverbs that do not introduce an external perspective (like swiftly) allow set-induction, while other POV-marked adverbs (like unfortunately) do interfere, cf. (38).

(38) a. Only I swiftly got a question that I could answer.

b. ?? Only I unfortunately got a question that I could answer.

We can represent the workings of the analysis in the following sketch of a tree:

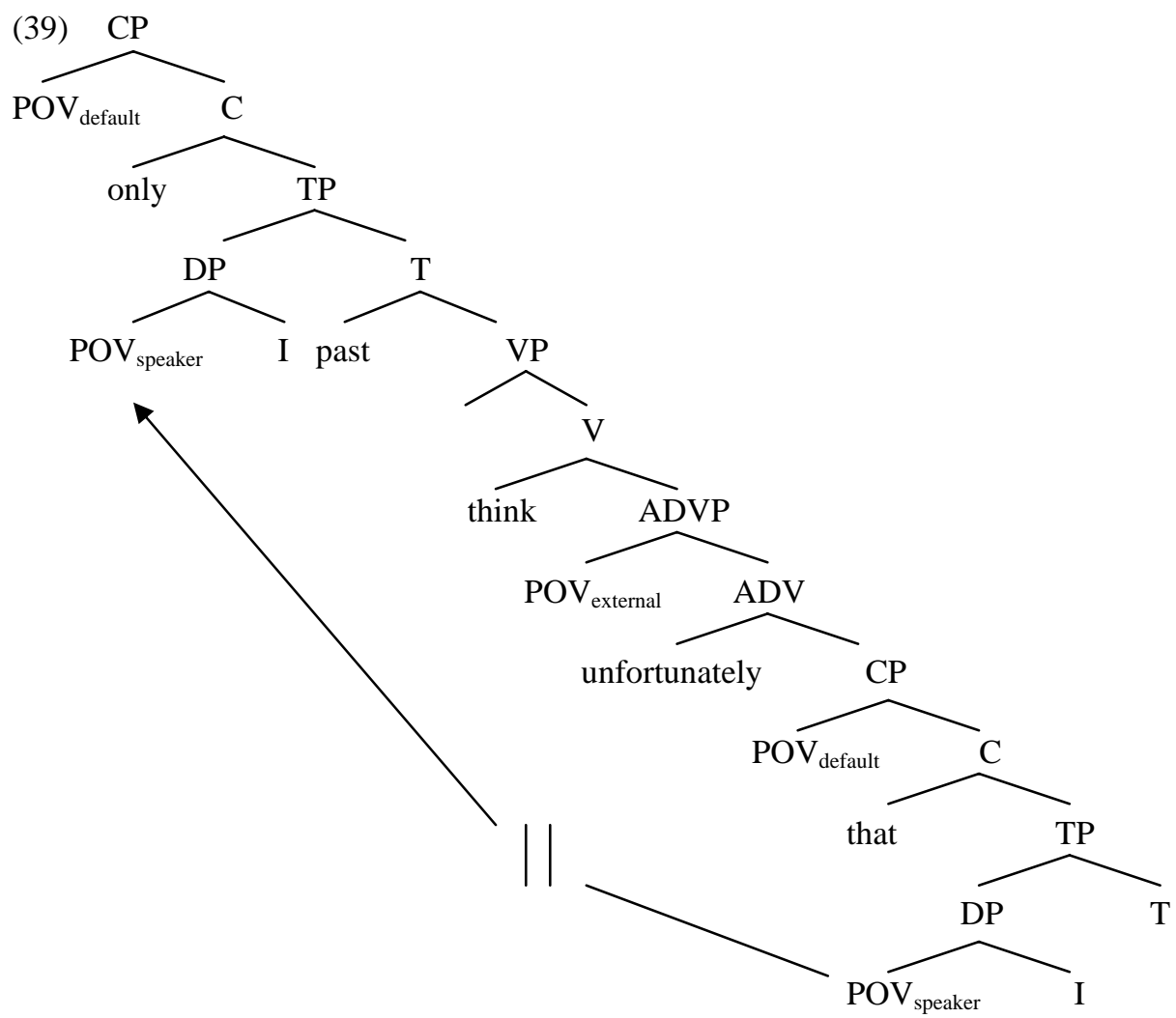


The POV-Ext of unfortunately will fail to be matched when the DP with a different Spec moves through the ADV-P Spec, either via adjunction of DP or by percolation of the POV-index. We leave out a full specification which engages other issues.

\subsection{Other cases}

Now we will indicate the direction of explanation for the other cases. The expressives likewise have a special ultra-internal POV, coming from an emotional sphere that is different from the intellectual one. The dative cases, likewise, suggest a second level, locative in Brandt's account, which is distinct from the primary level. And the sequence of tense cases suggest that the tense marker has a POV feature that is independently anchored in a second time dimension. All of these can be represented on the relevant Spec nodes and will produce an intervention if marked [+Distinct]. The consequence of the intervention is to block association with focus, which leaves a grammatical sentence that has not been linked. In a sense, then, the POV concept becomes, in its syntactic version, much like gender, which has been grammatized in a way that may not fully match its intuitive origins.

\subsection{Conclusion}

We have presented a new range of empirical data that expands what the focal binding linked to only-I involves. We have deliberately avoided a commitment to many aspects of the interfaces that link syntax, semantics, and the intentional system in order to seek a common representation for diverse information at the syntactic level. Needless to say, there are many respects in which the account needs refinement-it remains thus a sketch about how a notion of point of view can play a role in an efficient syntax and begin to build an appropriate interface.

\section{References}

Bhatt, R. and Pancheva, R., 2004. Late merger of degree clauses. Linguistic Inquiry 35:1-45.

Brandt, P., 2006. Receiving and perceiving datives (cipients): A view from German. In: Hole, D., Meinunger, A. and W. Abraham (eds.), Datives and Other Cases: Between argument structure and event structure, 103-139. Amsterdam: Benjamins.

Hardt, D., to appear. VP ellipsis and constraints on interpretation. In: Johnson, K. (ed.), Topics in Ellipsis. Cambridge: Cambridge University Press.

Heim, I., 1991. The first person. Class handout, MIT.

Heim, I., 2005. Features of pronouns: the semantics of number, gender and person. Talk given at the École normale supérieure, Paris. 
Hollebrandse, B., 2000. The Acquisition of Sequence of Tense. PhD dissertation, University of Massachusetts, Amherst.

Hornstein, N. J. Nunes, and K. Grohmann, 2005. Understanding Minimalism. Cambridge: Cambridge University Press.

Kratzer, A., 2006. Minimal pronouns. Talk given at the workshop QP structure, Nominalizations, and the Role of DP. Saarbrücken: Universität des Saarlandes.

Kratzer, A., 1998. More structural analogies between pronouns and tenses. In: D. Strolovitch \& A. Lawson (eds.), Proceedings of SALT VIII, 92-110. Ithaca: Cornell University.

Lebeaux, D. 2000. Language Acquisition and the Form of the Grammar. Amsterdam: Benjamins.

Nevins, A and Pranand, 2002. Shifty operators in changing contexts: indexicals in Zazaki and Slave. In: Young, R. (ed.), Proceedings of SALT XIV, 20-37. Ithaca: Cornell University.

Nunes, J. and E. Thompson, 1995. The discourse representation of temporal dependencies. In: Bertinetto, P.M., Bianchi, M., Higginbotham, J. and M. Squartini (eds.), Temporal Reference, Aspect, and Actionality, vol. I: Semantic and Syntactic Perspectives, 365-79. Torino: Rosenberg and Sellier.

Partee, B., 1989. Binding implicit variables in quantified contexts. In: C. Wiltshire, B. Music and R. Graczyk (eds.), Papers from CLS 25, 342-65. Chicago: Chicago Linguistics Society.

Potts, C., 2005. The Logic of Conventional Implicatures. Oxford: Oxford University Press.

Roeper, T., 1999. Notes on the Only I phenomenon. Manuscript, University of Massachusetts, Amherst.

Rooth, M., 1992. A theory of focus interpretation. Natural Language Semantics 1:75116.

Schlenker,P., 2003. A plea for monsters. Linguistics and Philosophy 26:29-120.

Speas, M., 1999. Person and point of view in Navajo direct discourse complements. In: Jelinek, E. (ed.), WCCFL Papers in Honor of Ken Hale, Cambridge, Mass.: MIT Press.

Speas, M., 2004. Evidentiality, logophoricity and the syntactic representation of pragmatic features. Lingua 114:255-276.

Speas, M., and C. Tenny, 2003. Configurational properties of point of view roles. In: A. Di Sciullo (ed.), Asymmetry in Grammar, 315-344. Amsterdam: John Benjamins.

Terada, A., 2003. A Study of Text Mining Techniques Using Natural Language Processing. $\mathrm{PhD}$ dissertation, Tokyo Institute of Technology. 